\title{
Laser-induced fluorescence measurement of the dynamics of a pulsed planar sheath
}

\author{
M. J. Goeckner and Shamim M. Malik \\ Engineering Research Center for Plasma Aided Manufacturing, The University of Wisconsin, \\ Madison, Wisconsin 53706-1608
}

\author{
J. R. Conrad \\ Department of Nuclear Engineering and Engineering Physics, The University of Wisconsin, Madison, \\ Wisconsin 53706-1687 \\ R. A. Breun \\ Engineering Research Center for Plasma Aided Manufacturing, The University of Wisconsin, Madison, \\ Wisconsin $53706-1608$
}

(Received 1 October 1993; accepted 17 December 1993)

\begin{abstract}
Using laser-induced fluorescence (LIF) the ion density near the edge of an expanding plasma sheath has been measured. These measurements utilized a transition of $\mathrm{N}_{2}^{+}$[the $\mathrm{P} 12$ component of the $X^{2} \Sigma_{g}^{+}(v=0) \Rightarrow B^{2} \Sigma_{u}^{+}(v=0)$ band] in a $N_{2}$ plasma. The strength of the laser-induced fluorescence was used as a measure of the temporally and spatially varying ion density. The expanding sheath was produced by applying $\mathrm{a}-5 \mathrm{kV}$ pulse to a polished planar electrode in the plasma source ion implantation device [J. R. Conrad et al., J. Vac. Sci. Technol. A 8, 3146 (1990)]. The laser beam was aligned normal to the surface and was reffected off the center of the electrode. The LIF diagnostic used here is nonperturbing whereas previous researchers have used Langmuir probes, which perturb the plasma, to make their measurements. As such, the data reported here represent a benchmark measurement of pulsed sheaths and allow a better comparison between experimental measurements and theoretical predictions. It has been found that the sheath edge moves approximately 16 times faster than the ion-acoustic velocity during the early part of the pulse, $t<1 \mu \mathrm{s}$, and then slows to approximately the ion-acoustic velocity after $6 \mu \mathrm{s}$. In addition to the LIF measurements, a biased probe was used far from the cathode to determine the sheath edge location. Good agreement is found when the LIF and probe data are compared. The LIF data also are compared to the predictions of a simulation that is based on a time-varying two-fluid model of the sheath [G. A. Emmert and M. A. Henry, J. Appl. Phys. 71, 113 (1992)]. While the predictions of the model show moderate agreement with the data, substantial discrepancies are observed. These discrepancies are attributed to a number of physical phenomena that are not included in the present model.
\end{abstract}

\section{INTRODUCTION}

A sheath is an electric field that provides a boundary between a plasma and an object. The sheath confines the more mobile species in the plasma and accelerates the less mobile species out of the plasma. For the typical case, in which the electrons are more mobile than the positively charged ions, the electric field points toward the object. This basic problem of plasma flowing into a wall is important, and has been studied since the inception of plasma physics. $^{1}$

Many models have been developed to describe sheaths. On the basis of on his experimental work, Langmuir was one of the first to model properly plasma sheaths. ${ }^{l}$ Other models of sheaths include, for example, the theory of Langmuir probes, ${ }^{2}$ and models of divertor plates in tokamaks. ${ }^{3}$ These models range from simple analytical expressions, such as Child's Law, ${ }^{4}$ to complex kinetic simulations. ${ }^{5}$ Models have been used to predict how different physical processes influence sheaths. For example, if the potential at the wall is made to vary in time, then the electric field in the sheath will also vary in time. ${ }^{6-9}$ Magnetic fields ${ }^{10}$ and collisions ${ }^{11}$ can also modify the sheath.
Since Langmuir's experiments, many other measurements have been reported, including several that involved in situ characterization of the sheath. Goldan ${ }^{12}$ measured the electric field in a planar DC sheath using an electron beam. Cho et al. ${ }^{13}$ measured the potential in a DC sheath using an emissive probe and used the same technique to examine the temporal response of the sheath potential to step-like and radio-frequency changes in the bias applied to the electrode. Winder et al. ${ }^{9}$ and later Shamim et al..$^{14-16}$ used biased probes to measure the dynamics of the edge of pulsed sheaths. A number of researchers have also used laser-induced fluorescence (LIF) to examine sheaths. Gottscho et al. ${ }^{17}$ measured the time-dependent ion density in a radio-frequency sheath. Gottscho and Mandich ${ }^{18}$ measured the electric field in a radio-frequency sheath. Finally, Goeckner et $a l .{ }^{19}$ measured the ion density and drift velocity in a DC sheath.

Of particular interest is the temporal response of the sheath to step-like potentials. ${ }^{8,9,13-16}$ Such pulsed sheaths have many uses. These range from basic plasma experiments, ${ }^{9,20}$ e.g., the excitation of ion-acoustic waves, to commercial applications, ${ }^{21}$ e.g., plasma source ion implantation. Many of the early articles on this subject dealt 
with basic plasma experiments and reported using potentials of approximately $-100 \mathrm{~V}$. In contrast, many of the more recent articles deal with commercial applications such as implantation of ions into semiconductors ${ }^{22}$ or steel tools. ${ }^{23}$ Typically for the implantation process, researchers use potentials ranging from -1 to $-100 \mathrm{kV}$. with respect to the grounded chamber.walls.

In this article we report LIF measurements of the propagation of a pulsed sheath that has been created by a high-voltage step-like potential to a cathodic planar electrode or ion target. To our knowledge this is the first time an LIF measurement of any pulsed sheath has been reported. The LIF technique provides a nonperturbing and in situ measurement of the plasma ions. This diagnostic technique has major advantages over many other in situ techniques. For example, when a biased Langmuir probe is located near the cathode, catastrophic arcing can occur between the probe and the target. LIF also allows full three-dimensional spatial resolution and accurate temporal resolution. This is in contrast to other nonperturbing diagnostic techniques, such as optical emission measurements, which are chord averaged and also typically time averaged.

We compare the LIF measurements to the predictions of a simple collisionless two-fluid model ${ }^{8,9}$ of pulsed plasma sheaths. This model has been used to predict the sheath propagation and ion energies in both scientific and commercial applications. In Sec. II we briefly review two-fluid theory as applied to plasma sheaths: In Sec. III we describe the experimental apparatus and the LIF diagnostic technique. In Sec. IV we compare our experimental results to the predictions of fluid theory and find moderate agreement at locations close to the electrode while at large distances the agreement deteriorates. We also compare our experimental results to those obtained with low-voltage pulses and find strong similarities. In doing so, we tie together the physics of the low-voltage and high-voltage pulsed sheaths. Finally, in Sec. V we summarize our results.

\section{TWO-FLUID SHEATH MODEL}

Two-fluid theory is widely ${ }^{24}$ used to model plasma sheaths. A number of simplifying assumptions are usually made when one applies this theory. First, the ions are assumed to be cold. Second, it is assumed that there is no impediment to the flow of electrons to the electrode surface. Third, it is typically assumed that the sheath region is source-free and collisionless. Thus, ions obey the equation of continuity

$$
\frac{\partial n_{i}}{\partial t}+\frac{\partial}{\partial x} n_{i} v_{i}=0
$$

and the equation of motion

$$
\frac{\partial^{2} x}{\partial t^{2}}=\frac{\partial v_{i}}{\partial t}=-\frac{e}{M} \frac{\partial}{\partial x} \phi(x, t) .
$$

Here, $M$ is the ion mass, $n_{i}(x), x$, and $v_{i}(x)$ are the ion density, position, and velocity in the sheath, $n_{0}$ and $v_{0}$ are the plasma density and ion velocity at the sheath-plasma boundary, $e$ is the electron charge, and $\phi(x)$ is the sheath potential relative to the plasma potential. Fourth, the electrons are assumed to be in thermal equilibrium and thus obey the Boltzmann relation

$$
n_{e}(x, t)=n_{0} \exp \left[e \phi(x, t) / T_{e}\right] ;
$$

where $n_{e}(x)$ and $T_{e}$ are the electron density and temperature. (In this article, all temperatures are given in units of energy.) Finally, Poisson's equation is obeyed

$$
\frac{d^{2} \phi}{d x^{2}}=-\frac{e}{\epsilon_{0}}\left(n_{i}-n_{e}\right)
$$

where $\epsilon_{0}$ is the permittivity constant.

Both Widner et al. ${ }^{9}$ and Emmert and Henry ${ }^{8}$ employed this model to examine the dynamics of pulsed sheaths. In particular, Emmert and Henry used Eq. (2) to advance elemental fluid cells one time step with a leapfrog algorithm. The densities of the cells were then updated using Eq. (1). Finally, the electric potential was determined from a linearized form of Eq. (4) by an iterative process. (Boltzmann's relation was used implicitly in the linearized form of Poisson's equation.)

When used in conjunction with commercial applications, this model can be used in a two-step process to predict both the depth and dose of ions implanted in the electrode. $^{23}$ This is accomplished by monitoring both the number of ions striking the surface and the profile of those ions' energy distribution. This information can then be passed to a Monte Carlo code that simulates the physical phenomena inside the electrode.

Unless otherwise noted, all theoretical predictions in this article were obtained with the simulation written by Emmert and Henry. ${ }^{8}$

\section{EXPERIMENT}

The LIF diagnostic was used to measure the timevarying ion density, and the position of the time-varying sheath edge. Throughout this article the time $t$ is relative to the start of the pulse and the position $x$ is relative to surface of the electrode. The edge of the sheath is defined as the position at which $n_{i}$ drops to one-half of the local plasma density before the pulse.

The Langmuir probe was also used to measure the position of the time-varying sheath edge. For this diagnostic technique, we define the sheath edge to conform to the definition used by Emmert and Henry. ${ }^{8}$ Under this definition, the sheath edge is the position at which $n_{e}$ drops to one-tenth of the local plasma density before the pulse.

The two diagnostic techniques provide differing measures of the sheath edge. Because the two techniquies measure different plasma parameters, notably $n_{e}(x, t)$ and $n_{i}(x, t)$, the definitions of the sheath edge cannot be the same. However, using the two-fluid model of direct current (DC) sheaths, ${ }^{19}$ one can show that these positions differ by approximately two Debye lengths. (The definition used with the probe data will place the edge closer to the target.) For the discharge parameters in the present experiment, this difference is $<0.2 \mathrm{~mm}$. 

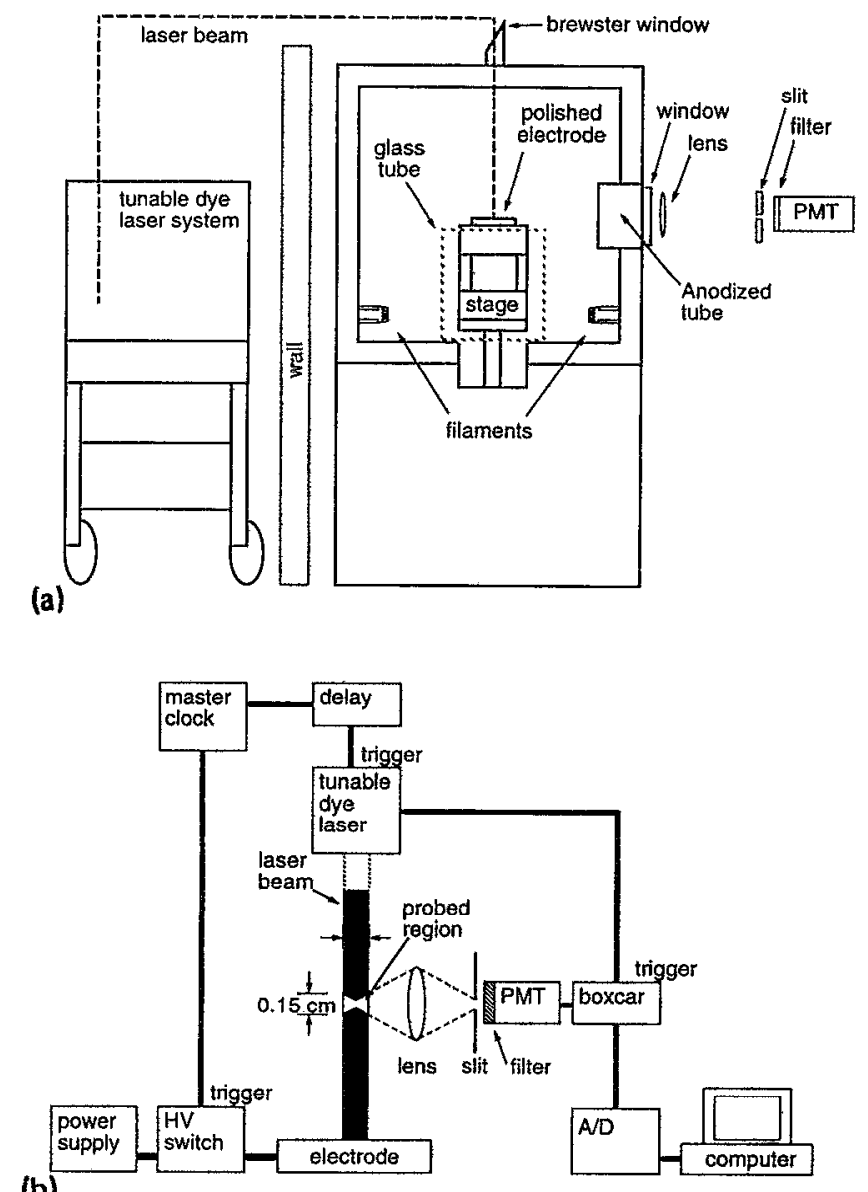

(b)

FIG. 1. Experimental setup for the laser-induced fluorescence (LIF) experiment. (a) The physical layout of the major components to the experiment. The components are the tunable dye laser system, the plasma chamber/electrode system, and the optical detection system. The detection optics include a $25.0 \mathrm{~cm} \mathrm{fl}, 5.08 \mathrm{~cm}$ diam glass lens, a razor blade slit, an interference filter, and a photomultiplier tube (PMT). Optical noise was reduced by an interference filter, bandwidth $10 \mathrm{~nm}$, center $390 \mathrm{~nm}$, positioning the filaments out of the line of sight, using the black anodized aluminum tube to reduce scattered light. Langmuir probe scans were also made along the same axis as the LIF scans. The probe was one sided and oriented to face the electrode. To use the probe, the Brewsier window was replaced with a sliding seal and the probe was inserted through the seal. (b) The data acquisition system for the LIF diagnostic. The pulsed sheath is created by switching the electrode from ground to a high negative voltage. By delaying the laser trigger, we could scan the timing of the laser shot throughout the high-voltage (HV) pulse. The location of the probed region was scanned by using a micrometer to move the slit, filter, and PMT as a unit. The shape of the probed region is exaggerated.

The experimental apparatus consisted of three major components, the plasma chamber, the LIF diagnostic, and the pulsed electrode. The general layout of these components is shown in Fig. 1(a).

\section{A. Plasma chamber}

The plasma chamber shown in Fig. 1(a) is cubical with an interior width of $85 \mathrm{~cm}$. Most of the plasma was produced and sustained by primary electrons emitted from 16 thoriated-tungsten filaments. These filaments were positioned out of the direct view of the LIF detection system. To increase the emissivity of the filaments, they were resistively heated. A bias between the filaments and the chamber accelerated the electrons off the filaments and into the plasma. This acceleration provided the primary electrons with sufficient energy to ionize the neutral gas. Magnets, arranged in a line-cusp geometry, ${ }^{25}$ were used to increase the confinement of the primary electrons and to some extent, the plasma. Besides the primary electrons, other high energy electrons were also present in the discharge. These were secondary electrons, which resulted from high energy ions bombarding the electrode. While it is likely that some of the plasma was produced by the secondary electrons, they were not present in sufficient numbers to maintain the discharge (see the Appendix). A complete description of the device is given in Ref. 23. In a similar discharge, Goeckner et $a i^{26}$ have shown that the translational temperature of the ions is comparable to the chamber wall temperature.

\section{B. Electrode}

The electrode is a $12.7 \mathrm{~cm}$ diam, $1.27 \mathrm{~cm}$ thick stainless steel disk. One side of the disk has been polished to a mirror finish. The electrode is positioned in the center of the chamber. The residual magnetic field is small in this region, $<10 \mathrm{G}$, and it is randomly oriented with respect to the electrode surface. As shown in Fig. 1, the electrode sits on a $17.78 \mathrm{~cm}$ diam oil cooled stage that is electrically insulated from the chamber. While most of the stage is also constructed of stainless steel, the top most portion is a 0.95 $\mathrm{cm}$ thick, $17.78 \mathrm{~cm}$ diam plate of aluminum. Silver paint was used to provide definite electrical contact between the stage and the electrode. A glass tube (Borosilicate, $24 \mathrm{~cm}$ outer diam, $0.9 \mathrm{~cm}$ wail, $40.6 \mathrm{~cm}$ long) was used to shield the sides of the stage from the plasma and thus reduce arcing to the chamber walls. In this configuration, only the aluminum plate and the stainless steel electrode were exposed to the plasma.

The sheath was pulsed by switching the bias of the electrode to a large negative voltage. A square wave generator (Global specialists 4001) was used to drive the highvoltage (HV) switch (Thompson TH5188). The width of the supplied square wave set the length of the HV pulse. The HV switch would close approximately $2.2 \mu$ s after the trigger, with a jitter of $0.1 \mu \mathrm{s}$. The switching time ${ }^{14}$ for the HV pulse is typically $10 \mathrm{kV} / \mu$ s with a lower limit of approximately $1.9 \mu \mathrm{s}$.

\section{LIF diagnostic}

LIF is a widely used plasma diagnostic technique. This technique can be described as follows: Laser light slowly scanned in wavelength $\lambda_{1}$ is fired into the plasma. When $\lambda_{1}$ is in resonance with an optical transition of the ions, $\lambda_{i}$, laser photons are absorbed. Most of the energy absorbed by the ions will subsequently be emitted as fluorescence photons. ${ }^{19,27}$ The number of fluorescence photons emitted is proportional to the density of ions in the appropriate energy state; and for low laser intensities, it is also proportional to the intensity of the laser beam. ${ }^{27}$ (To correct for variations in the laser beam's intensity, it was monitored and the data were numerically corrected after the scans 
were completed. ${ }^{19,26}$ ) One usually finds $\lambda_{i}$ by scanning $\lambda_{1}$ while measuring the resulting fluorescence intensity. On completing the scan, one finds a curve, or measured spectral line, that varies with $\lambda_{1}$. This measured spectral line is a convolution of the laser's spectral line and the ion's spectral line. For broadband lasers with moderate power levels, such as the one employed for this experiment, the bandwidth of the measured line is representative of the laser's linewidth while the peak height is representative of the number of ions in that energy state. ${ }^{28}$

The layout of optical systems and the electrode are shown in Fig. 1(b). The laser system used for the LIF measurements consisted of a pump laser (Lumonics EX400) and a tunable dye laser. The pump laser was set up to lase at $337 \mathrm{~nm}$ and it has a pulse duration of $15 \mathrm{~ns}$. The dye laser system is similar in construction to the laser reported by Lawler, Fitzsimmons, and Anderson. ${ }^{29}$ The laser is both grating and pressure tuned. Course tuning is accomplished by changing the angle of the grating. Fine tuning is accomplished by changing the density of $\mathrm{N}_{2}$, and thus the index of refraction, around the grating. This density is increased by bleeding $\mathrm{N}_{2}$ from a gas cylinder into a pressurized housing that encloses the grating. While extracavity etalons are often used to narrow a laser's bandwidth, ${ }^{29}$ one was not used in this experiment and as such the laser bandwidth was $0.0046 \mathrm{~nm}\left(0.3 \mathrm{~cm}^{-1}\right)$. Finally, using pressure tuning, the minimum step size of $\lambda_{1}$ is $0.00016 \mathrm{~nm}$. However, for this experiment we used a step size of $0.0008 \mathrm{~nm}$. These step sizes correspond to changing the pressure inside the grating's housing by 1 and 5 Torr, respectively.

The operating wavelength of the laser was found and set by using a scanning monochrometer. The monochrometer (Thermo Jarrell Ash 82020), which is not shown in Fig. 1, is $0.5 \mathrm{~m}$ long and has a resolution of approximately $5 \AA$. It was positioned on the side of the plasma chamber opposite from the detection optics. A lens was used to focus emission from the plasma onto the entrance slit of the monochrometer. Without the laser beam present, the monochrometer was scanned to find the appropriate spectral line from the $\mathrm{N}_{2}^{+}$ions. The plasma was then extinguished and the laser beam was introduced into the vacuum chamber. A small wire was inserted into the beam path and laser light was scattered into the entrance slit of the monochrometer. The laser's grating was then adjusted to tune the laser to approximately the same wavelength as that of the spectral line from the $\mathrm{N}_{2}^{+}$ions.

The laser beam was aimed to strike at normal incidence, near the center of the electrode. The diameter of the beam was $0.7 \mathrm{~cm}$ at the electrode surface. The electrode was highly polished and the laser exited the plasma chamber down the same route it had entered. The laser was fired once for each HV pulse.

As shown in Fig. 1(b), the detection optics were positioned to view the sheath at right angle to the direction of the laser beam. A lens and slit arrangement was used to determine the volume viewed by the detection system. The $25.0 \mathrm{~cm}$ focal length, $5.08 \mathrm{~cm}$ diam glass lens was used to focus fluorescence from the plasma on to a $0.1 \mathrm{~cm}$ wide slit.
The lens and slit were positioned to give a magnification of 0.9 .

The intersection of the volume viewed by the detection optics and the laser beam defined the probed volume. Because of the geometry of the experiment, the volume probed with the LIF diagnostic varied slightly from location to location $( \pm 20 \%)$. To numerically correct for this variation, Rayleigh scattering ${ }^{30}$ in atmospheric pressure $\mathrm{Ar}$ was used to determine the relative collection volume at each of the probed locations.

We used a variety of techniques to reduce optical noise. First, we employed an interference filter to stop most of the optical noise from reaching the photomultiplier tube (PMT). This filter (Rolyn 66.3325-390) had a $10 \mathrm{~nm}$ bandwidth and was centered at $390 \mathrm{~nm}$. Second, we eliminated most of the scattered laser light by using a $20.3 \mathrm{~cm}$ diam, $15.2 \mathrm{~cm}$ long, black anodized aluminum tube near the detection window. Third, we eliminated most of the light produced by the plasma, by electronically gating the signal from the PMT. For this experiment the gate had a width of $40 \mathrm{~ns}$ and was synchronized with the laser shot.

Figure 1(b) shows the major components of the data acquisition system. The PMT (Hamamatsu 1P28A) was used to detect the collected fluorescence, and it had a cathode voltage of $-1200 \mathrm{~V}$. The current signal from the PMT was acquired with the use of a boxcar averager (Stanford Research SR250). The boxcar was set to average over 10 HV pulses. Concurrently, a gauge (MKS Baratron 220CA) was used to monitor the pressure inside the laser's grating housing. This information was then passed via an analog-to-digital board (Computer Boards Inc. CIO$\mathrm{AD} 16$ ) to a computer (Computer Components-486 processor). After a scan was completed, all the LIF data for a given laser pressure, and hence $\lambda_{1}$, was digitally averaged. The number of laser shots averaged over depended on the rate of the HV pulses and the rate at which the laser's pressure changed. For this experiment we averaged over approximately 150 shots for each $\lambda_{1}$.

A similar arrangement was used by Goeckner et al. ${ }^{19}$ to measure both $n_{i}(x)$ and $v_{i}(x)$ in a DC plasma sheath. However, the bandwidth of the laser, $.0 .09 \mathrm{~cm}^{-1}$, in that experiment is much narrower than the bandwidth of the laser, $\approx 0.3 \mathrm{~cm}^{-1}$, used here. Because of this substantially larger bandwidth, direct measurement of the drift velocity was impractical in this experiment.

For this experiment, we used a transition of $\mathrm{N}_{2}^{+}$[the P12 component of the $X^{2} \Sigma_{g}^{+}(v=0)-B^{2} \Sigma_{u}^{+}(v=0)$ band] in an $\mathrm{N}_{2}$ plasma. At the resolution of our laser, the $\mathrm{P} 12$ and P13 components overlap. Throughout this article we refer to the sum of these components simply as the P12 component. One might wonder if this transition accurately represents the ions as a whole.: There are three main concerns.

The first concern that one might have is that nitrogen plasmas typically have both $\mathrm{N}_{2}^{+}$and $\mathrm{N}^{+}$ions. Tang et al. ${ }^{31}$ have shown in a similar discharge that $\mathrm{N}^{+}$represents $20 \%-40 \%$ of the total population of ions. This percentage depends on a number of parameters, including the gas pressure and plasma density ${ }^{31}$. (This percentage perhaps even depends on the configuration of the plasma chamber.) 
However, both ion species will be subject to the same electric field. By using conservation of flux and the mass ratio one could calculate one density profile from the other.

The second concern that one might have is that during the HV pulse secondary electrons are produced when the high energy ions strike the electrode surface. These secondaries are then accelerated back across the sheath and into the plasma. If the secondaries ionize a large number of neutrals, both the fraction of ions that are $\mathrm{N}^{+}$and the plasma can change during the pulse. For nitrogen ions having $5 \mathrm{keV}$ of energy, approximately 2.4 secondary electrons are produced for each ion striking a stainless steel surface, while approximately 7.2 are produced for an aluminum surface. ${ }^{32}$ Most of the secondary electrons gain 5 $\mathrm{keV}$ of energy as they fall down the sheath potential. This energy is markedly different from that of the primary electrons, which have energies of $125 \mathrm{eV}$. At these energies and $0.5 \mathrm{~m}$ Torr pressure, the mean free path of ionization of $\mathrm{N}_{2}$ by the primaries ${ }^{33}$ is $1541 \mathrm{~cm}$, while for the secondaries ${ }^{34}$ it is $276 \mathrm{~cm}$. However, the ratio of the production rates for $\mathrm{N}^{+}$and $\mathrm{N}_{2}^{+}$is approximately the same at both energies $\left(\mathrm{N}^{+}:: \mathrm{N}_{2}^{+} \Rightarrow 1:: 3.7\right)$ and thus the ratio of the ion species produced should not change. Also, because the pulse width is short compared to the lifetime of the ions, the secondary electrons do not significantly add to the plasma density. This is discussed in detail in the Appendix. The lack of substantial plasma production is confirmed by measurements in similar discharges, ${ }^{14-16}$ which do not indicate any change in the plasma density during the HV pulse.

The third concern one might have is that the rotational temperature of the $\mathrm{N}_{2}^{+}$ions might change during the $\mathrm{HV}$ pulse. The strength of the transition that we use to represent the density of the $\mathrm{N}_{2}^{+}$ions is dependent on the rotational temperature $T_{\text {rot }}$ as well as the density. (Because $\mathrm{N}_{2}^{+}$ is homonuclear, it will not exhibit any vibrational dipole transitions, and hence it will not have a vibrational temperature. ${ }^{35}$ ) We argue below (Sec. IV A) that $T_{\text {rot }}$ is substantially independent of the spatial and temporal location in the $\mathrm{HV}$ pulse. Finally, we note that all the $\mathrm{N}_{2}^{+}$ions are subject to the same electrical forces, regardless of the excitation state.

\section{Probe diagnostic}

We used a Langmuir probe in two diagnostic capacities. The probe is a one-sided $0.64 \mathrm{~cm}$ diam tantalum disc and was oriented to face the electrode. All the Langmuir probe scans were made along the same axis as the LIF scans. To use the probe, the Brewster window was replaced with a sliding seal and the probe was inserted through the seal.

Firstly, we used the Langmuir probe in the "standard" manner. ${ }^{2}$ The data collected in this manner were used to determine the plasma density $n_{0}$, the electron temperature $T_{e}$, the plasma potential, and the floating potential. All "standard" probe data were collected without the pulser on. Because of the long time between pulses, $\approx 1 / 30 \mathrm{~s}$, the plasma parameters before the $\mathrm{HV}$ pulse should be the same as those measured with the probe. In addition, as is dis-

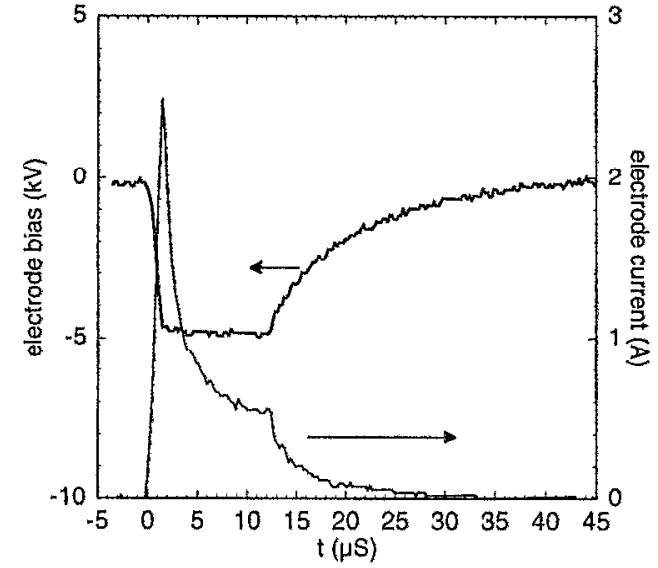

FIG. 2. Time-varying bias applied and current drawn to electrode. Most of the electrode current is due to secondary electron emission (Ref. 32). The time $t$ is given relative to the start of the HV pulse.

cussed in the Appendix, the plasma will be only marginally different with the pulser on and, as noted above, this is confirmed by previous experiments.

Secondly, the Langmuir probe was used to measure the position of the plasma sheath edge. These measurements were made in the same manner that Winder et al. ${ }^{9}$ and Shamim et al. ${ }^{14-16}$ made their measurements. For this experiment the probe was biased to $72 \mathrm{~V}$ and the electron current drawn to the probe was monitored. This bias produced a $0.29 \mathrm{~cm}$ wide sheath around the probe. (This sheath width is based on a space-charge limited flow of electrons from the plasma.) The positions of the Langmuir probe were corrected for the probe's sheath width. Shamim et al. ${ }^{14-16}$ have shown that the profile of the time-varying current to the probe is independent of the applied bias. The current collected by the probe is from the following: the local electron density $n_{e}$, the secondary electrons from the electrode, and the displacement current. When the sheath passes the location of the probe, the collected current drops because $n_{e}$ drops. As noted above, for this diagnostic technique we defined the arrival of the sheath edge as the time at which $n_{e} \approx 0.1 n_{0}$. This definition was used to conform to the definition used in the simulation by Emmert and Henry. ${ }^{8}$

\section{RESULTS AND DISCUSSION}

For this experiment, the pressure was $0.5 \mathrm{~m}$ Torr while the discharge current and voltage were $2.0 \mathrm{~A}$ and $-125 \mathrm{~V}$. The HV pulse was fired at a rate of $30 \mathrm{~Hz}$. During the pulse, the voltage fell linearly from ground to $-5 \mathrm{kV}$ over the first $1.9 \mu \mathrm{s}$. This voltage was held for $11 \mu \mathrm{s}$, and then it exponentially decayed back to ground over the next $25 \mu \mathrm{s}$. An example of the pulse shape is shown in Fig. 2. Without the high-voltage pulser on, we found that for the $x=4.0$ to $10.0 \mathrm{~cm}$ range, $T_{e}=0.57 \pm 0.01 \mathrm{eV}, n_{0}=6.25 \pm 0.7 \times 10^{9}$ $\mathrm{cm}^{-3}$, the plasma potential was $0.92 \pm 0.06 \mathrm{~V}$, and the floating potential was $-14.8 \pm 5.2 \mathrm{~V}$. We expect the plasma potential to decrease and the plasma density to increase during the HV pulse. The plasma potential should 


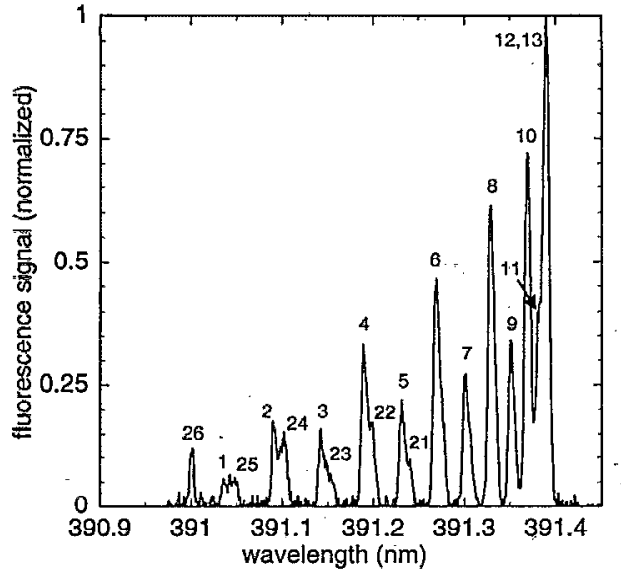

FIG. 3. The $P$ branch of the $\mathrm{N}_{2}^{+} X^{2} \Sigma_{g}^{+}(v=0)-B^{2} \Sigma_{u}^{+}(v=0)$ band. These data were collected from $3.6 \mathrm{~cm}$ above the electrode, $1 \mu \mathrm{s}$ before the HV pulse was applied to the electrode. The number labeling corresponds to the rotational quantum number $J$ of the lower energy state of the electronic transition. These data are used in Fig. 4 to determine the rotational temperature $T_{\text {rot }}$ of the $\mathrm{N}_{2}^{+}$ions.

decrease because the pulse serves to enhance the loss rate of the ions. To balance this, the loss rate of the electrons must also increase and hence the plasma potential should drop. Such drops have been observed by Collins and Tendys. ${ }^{36}$ The plasma potential, however, should not fall below ground, as the electrons would then quickly leave the plasma and quasineutrality would be violated. Finally, as discussed in the Appendix, the plasma density should increase approximately $1 \%$ during the HV pulse.

The LIF data were gathered in the following manner: The position of the lens and slit determined the location that was observed in the sheath. For each measured position and time in the sheath, the wavelength of the laser was scanned by changing the pressure in the grating housing. Wavelength scans were performed for a series of times at a given location. After the sheath edge was observed to pass a location, the detection optics were aligned to observe a location further from the electrode and the process was repeated.

To fully validate the experimental data, we must show that the LIF signals are an appropriate measure of the ions as a whole. Our LIF measurement is of the strength of the P12 component of the $X^{2} \Sigma_{g}^{+}(v=0)-B^{2} \Sigma_{u}^{+}(\nu=0)$ band of $\mathrm{N}_{2}^{+}$. For the strength of this spectral line to represent accurately the ions as a whole, both $T_{\text {rot }}$ and the $\mathrm{N}^{+} / \mathrm{N}_{2}^{+}$ density ratio must be approximately constant, both temporally and spatially, throughout the region examined. Above, we have argued that the density ratio should be constant. Below, we argue that $T_{\text {rot }}$ is constant.

\section{A. Rotational temperature $T_{\text {rot }}$}

The rotational temperature $T_{\text {rot }}$ of the $\mathrm{N}_{2}^{+}$ions was determined to be $0.047 \mathrm{eV}$ ( $543 \mathrm{~K}$ ) by measuring the intensity of the $\mathrm{P}$ branch of the $X^{2} \Sigma_{g}^{+}(v=0)-B^{2} \Sigma_{u}^{+}(v=0)$ band of $\mathrm{N}_{2}^{+}$at $t=-1 \mu \mathrm{s}, x=3.6 \mathrm{~cm}$. These data are shown in Fig. 3. The normalized intensity of the $J$ th component of the band, $I_{J}$, is related to $T_{\text {rot }}$ by ${ }^{35}$

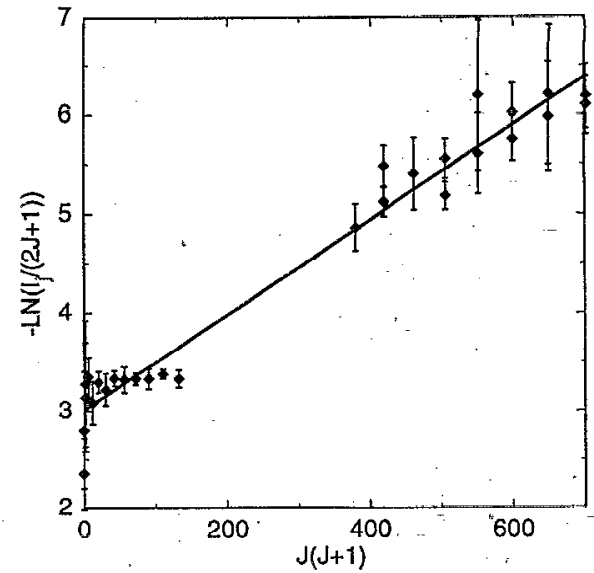

FIG. 4. Plot of $L N\left[I_{J} /(2 J+1)\right]$ as a function of $J(J+1)$. Here, $I_{J}$ is the normalized intensity of the $P(J)$ branch. By fitting a straight line to this graph, $T_{\text {rot }}$ was determincd to be $0.0429 \mathrm{eV}$. The Pauli exclusion principle was invoked in the normalization.

$$
I_{J} \propto(2 J+1) \exp \left(\frac{-B h c J(J+1)}{T_{\text {rot }}}\right) .
$$

Here, $J$ is the rotational quantum number associated with the lower energy state; $B\left(\approx 2 \mathrm{~cm}^{-1}\right)$ is a rotational constant $^{37}$ of the ion, $h$ is Planck's constant, and $c$ is the speed of light. (Variation in the component strength due to the Pauli exclusion principle is accounted for in the normalization of $I_{J}$.) As is shown in Fig. 4, the rotational temperature is found from these data by plotting $-L N\left[I_{J} /(2 J+1)\right]$ vs $J(J+1)$ and then fitting a straight line to the graph. While one would not expect the translational temperature (Ref. 26) ( $\approx 0.03 \mathrm{eV}$ ) and $T_{\text {rot }}$ to be in thermal equilibrium, we find that they exhibit very similar values.

The LIF data indicate that $T_{\text {rot }}$ is constant at all the temporal-spatial locations examined. Any change in $T_{\text {rot }}$ would result in a change in the ratio between the strengths of the P12 and P10 spectral lines. Typically our LIF scans included both the P12 and P10 spectral lines. We have found that the ratio of the P10 to P12 line strengths is approximately 0.7 for all of our experimental data. The deviations from this value are typically \pm 0.1 and are random.

The ratio of the $\mathrm{P} 10$ to $\mathrm{P} 12$ line strengths can be used to estimate that variations in $T_{\text {rot }}$ cause less than a $10 \%$ error in the measured density. This is done using Eq. (5). One finds that for a ratio of $0.6, T_{\text {rot }}=0.037 \mathrm{eV}(445 \mathrm{~K})$. Likewise for ratios of 0.7 and 0.8 , one finds a $T_{\text {rot }}$ of 0.027 $\mathrm{eV}(320 \mathrm{~K})$ and $0.021 \mathrm{eV}(257 \mathrm{~K})$, respectively. These temperatures can then be used with Eq. (5) to examine the percentage of the total population of the $P$ band that is in the P12 state. One finds that the percentage is $8.6 \%$ for $T_{\text {rot }}=0.027 \mathrm{eV}$ and $10.3 \%$ for the other temperatures. Thus, one would expect less than a $20 \%$ error in the measured density if one ignored any possible variations in the measured value of $T_{\text {rot }}$.

This result is not surprising as one would not expect $T_{\text {rot }}$ to vary outside the sheath. Because momentum trans- 
fer is small in electron-ion collisions, and because ions colliding with the chamber walls will likely result in the production of neutral gas molecules, the only processes that can influence $T_{\text {rot }}$ are ion-neutral collisions and the production of hotter ions. First, one would not expect $T_{\text {rot }}$ to change as the result of ion-neutral collisions. It is likely that the neutrals are thermalized with the walls, so that the ion-neutral collisions cannot strongly influence $T_{\text {rot }}$. Additionally, one would not expect $T_{\text {rot }}$ to change except over distances greater than several mean free paths. At the neutral pressure used in this experiment, the mean free path for ion-neutral collisions ${ }^{38}$ is approximately $6.0 \mathrm{~cm}$. Our LIF measurements extended from $x=0.8$ to $4.7 \mathrm{~cm}$. Second, the secondary electrons emitted from the electrode may result in a brief increase in the production of ions. These "new" ions are produced by secondary electron impact on neutral molecules. However, these ions account for approximately $1 \%$ of the total ion population, see the Appendix. Thus, before the passage of the sheath edge, one would not expect large changes in $T_{\text {rot }}$ across the region probed.

In contrast, $T_{\text {rot }}$ might change considerably inside the sheath. Inside the sheath the ions will have large translational energies. If one of these high energy ions collides with a neutral, it is likely that some of the translational energy will be transferred to rotational energy. Large numbers of these collisions can thus completely change $T_{\text {rot }}$ inside the sheath. It has been shown that collisional drag will begin to degrade the translational energy of the ions in the sheath at distances greater than 0.53 times the mean free path. ${ }^{11}$ One can use this result to speculate that this is approximately the same distance at which $T_{\text {rot }}$ begins to change. For this experiment, this distance ${ }^{38}$ is $3.2 \mathrm{~cm}$. However, our LIF measurements were obtained no deeper than $0.5 \mathrm{~cm}$ inside the sheath.

For these reasons, we have assumed that $T_{\text {rot }}$ is the same at all the temporal-spatial locations examined with LIF. All of these locations were either in the bulk plasma or near the sheath edge. The LIF data obtained inside the sheath were always at distances that were less than $0.5 \mathrm{~cm}$ from the sheath edge. Thus, we expect $T_{\text {rot }}$ to be the same throughout the scanned regions.

\section{B. Ion density and sheath propagation}

\section{LIF measurements}

The strength of the LIF signal from the P12 component of the $X^{2} \Sigma_{g}^{+}(\nu=0)-B^{2} \Sigma_{u}^{+}(\nu=0)$ band of $\mathrm{N}_{2}^{+}$was used as a measure of the density of the ions. This is possible because both $T_{\text {rot }}$ and the ratio of the $\mathrm{N}^{+}$to $\mathrm{N}_{2}^{+}$densities are approximately constant during the HV pulse. The LIF signal has been normalized to compensate for variations in the laser intensity. ${ }^{27}$ In addition, we have scaled the LIF data taken at $t=-1 \mu \mathrm{s}$ to match the Langmuir probe measurements of $n_{0}$ in the $x=4.0$ to $5.0 \mathrm{~cm}$ range. This scaling is shown in Fig. 5 and is used on all subsequent LIF data.

Figure 6 shows $n_{i}$ as a function of both $x$ and $t$. As expected, there is no indication of additional plasma pro-

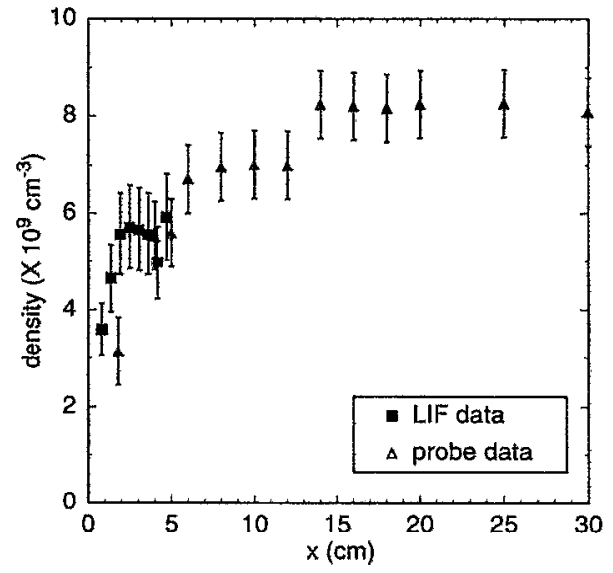

FIG. 5. The plasma density along the axis of the chamber. The position $x$ is the distance above the electrode. Because the LIF measurements of the ion density are in arbitrary units, we have scaled them to match the Langmuir probe measurement of the electron density in the $x=3.0-5.0$ $\mathrm{cm}$ range. This scaling is used throughout the article.

duction outside the sheath. We see in these data a number of phenomena. First, before the arrival of the sheath, $n_{i}$ increases as one moves away from the cathode surface. It is likely that this increase is a manifestation of the plasma presheath. Second, when the sheath edge arrives at a given location, $n_{i}$ plummets to approximately zero in a very short time. For example, at $x=1.37 \mathrm{~cm}$, the required time is approximately $0.3 \mu$ s or 0.94 ion plasma periods, $1 / f_{p i}$.

Figure 7 shows the experimentally measured position of the sheath edge as a function of $t$. Using our definition of the sheath edge, a drop of $50 \%$ in $n_{i}$, one sees from Fig. 6 that the sheath passes $x=1.37 \mathrm{~cm}$ at $t \approx 1.1 \mu \mathrm{s}$. If one were to use a slightly different definition of the sheath edge, for example, a $25 \%$ drop, one would determine that the sheath passed a given location at almost the same time.

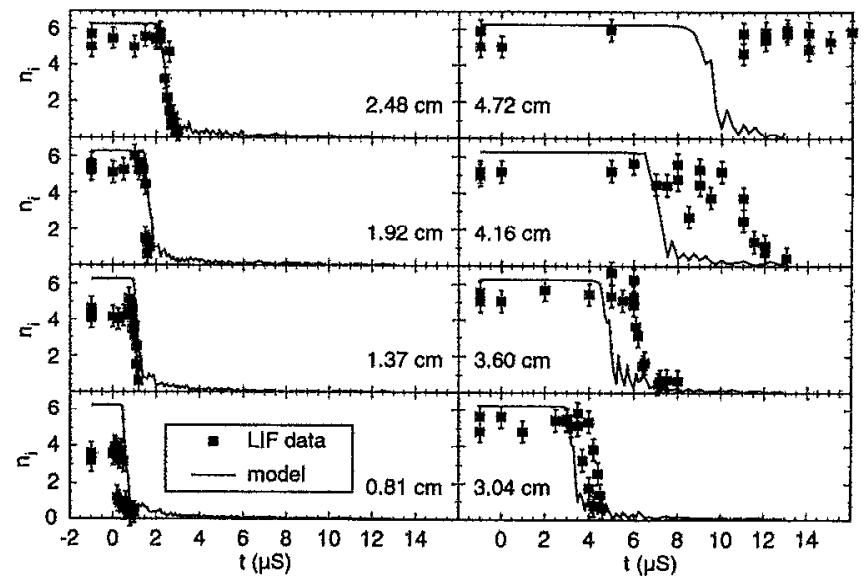

FIG. 6. LIF measured ion density compared to theoretical predictions. When one compares the time at which the ion density drops, the LIF data and the model's predictions show reasonable agreement near the electrode while poor to no agreement is found at large distances. Additionally, the model does not account for the existence of a presheath. A presheath is evident in the LIF data. 


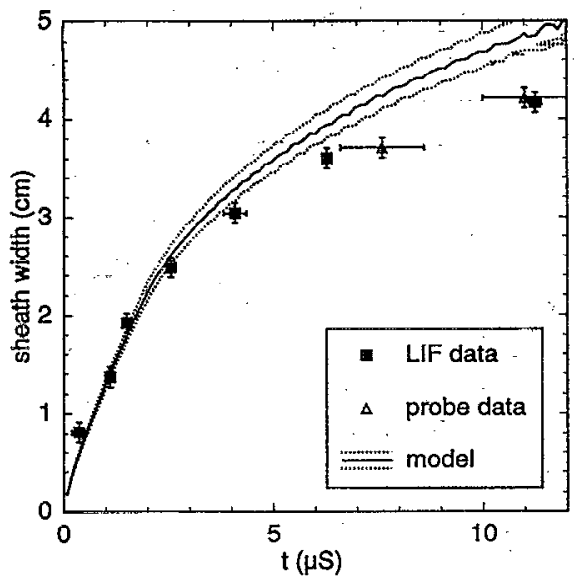

FIG. 7. Experimental and theoretical sheath edge positions as functions of $t$. The Langmuir probe data and the LIF data closely agree. As in Fig. 6 , the experimental data and the theoretical predictions show fair agreement near the electrode. This agreement deteriorates at large distances. The disagreements observed here and in Fig. 6 might be removed if the three physical phenomena discussed in the text are added to the model. Because of arcing, Langmuir probe data could not be obtained closer to the electrode. The positions of the Langmuir probe data were corrected for the probe's sheath width.

Figure 8 shows the sheath edge velocity, $V_{s}$ calculated from the data shown in Fig. 7. These velocities are calculated by dividing the change in position by the change in time and they are associated with a position halfway between the experimentally probed locations. We see that at small distances (early times) the sheath is moving faster than the ion-acoustic velocity $C_{s}$. At larger distances (longer times) the sheath moves at approximately $C_{s}$.

\section{Langmuir probe measurements}

A Langmuir probe was used to measure the time required for the moving sheath edge to pass a given location.

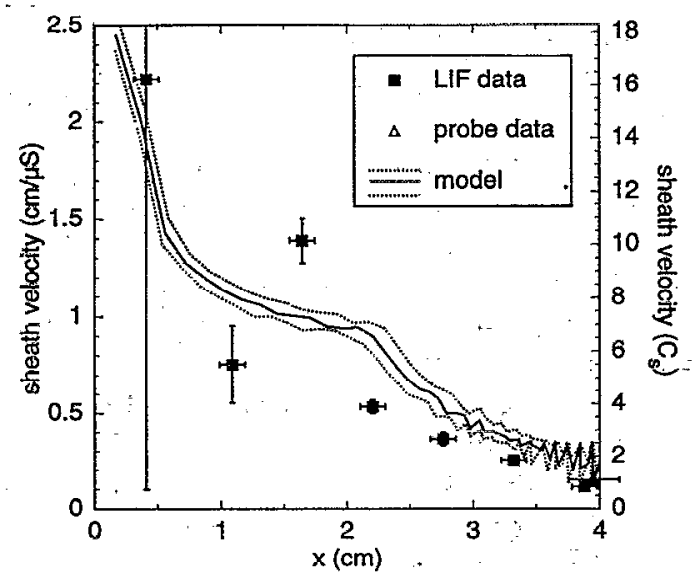

FIG. 8. Estimated sheath edge velocities $V_{s}$. The sheath edge velocity is given both in real units, $\mathrm{cm} / \mu \mathrm{s}$, and in units of the ion-acoustic velocity $C_{s}$. Note that at small distances (early times) the sheath is moving much faster than $C_{s}$. At larger distances (Ionger times) the sheath moves at approximately $C_{s}$. These results exhibit strong similarities to the results found for low-voltage (Ref. 9) pulsed sheaths.

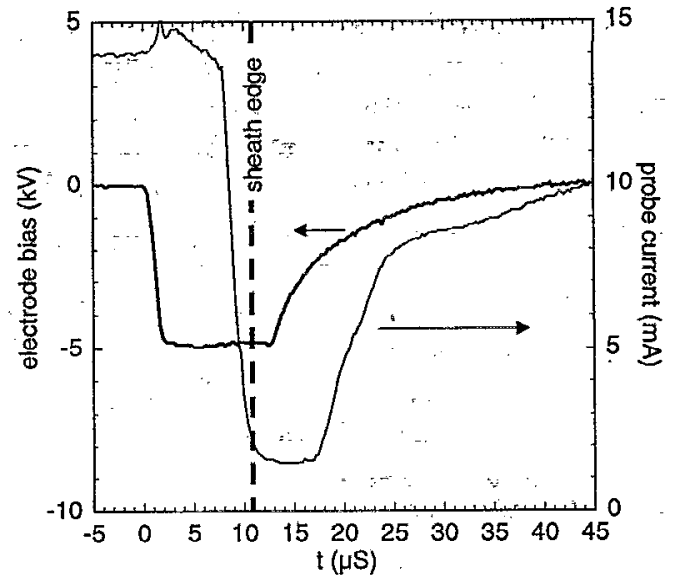

FIG. 9. Time varying bias applied to electrode and electron current drawn to the probe. Electron current was drawn to the probe by biasing it to $+72 \mathrm{~V}$. This current is the combination of current from the plasma, secondary electrons emitted from the electrode surface, and displacement current. We define the sheath edge to be the time when the electron current from the plasma goes to one-tenth its original value. Detailed analysis for similar traces is given in Refs. 14-16. For this scan; the probe was $4.5 \mathrm{~cm}$ above the electrode.

A typical example of the measured current during a pulse is given in Fig. 9. As discussed above, the measured electron current has the following features: For $t<0$, the current is constant. At $t \approx 0$, a brief displacement current appears and secondary electrons begin to add to the collected current. At some time after $t=0$, a sharp drop occurs when the sheath edge passes the probe. This leaves only the current due to the secondary electrons. Finally at times longer than the HV pulse, the current slowly rises as the sheath retreats.

Figure 7 shows the time varying position of the sheath edge as measured with the probe, while Fig. 8 shows the corresponding sheath velocity. It is seen that the probe data closely agree with the LIF data. Because of problems with arcing in similar experiments, ${ }^{14-16}$ probe data were not obtained near the electrode. These data serve to confirm the data obtained using LIF.

\section{Model predictions}

This experiment was designed to test the two-fluid model of HV pulsed sheaths that is reviewed in Sec. II. Toward this end the experiment was performed under conditions similar to those assumed in the model ${ }^{8}$ First, the translational ion temperature ${ }^{26}$ is approximately the same as chamber walls, $0.03 \mathrm{eV}$. The electron temperature is much higher than this value, ${ }^{, i} T_{e}=0.57 \mathrm{eV}$. Thus, approximating the translational ion temperature as zero is reasonable for this discharge. Second the magnetic field is less than $10 \mathrm{G}$ near the sheath. Thus, the electrons and ions are not magnetized. This is seen by noting that the Larmor radius to Debye length ratio is much greater than 1 for all charged species ( $\mathrm{N}^{+}$ions: $1825, \mathrm{~N}_{2}^{+}$ions: 2581 , bulk electrons: 49 , primary electrons: 735 , secondary electrons: 4648). Additionally, the magnetic field is randomly oriented at the electrode's surface and thus it will not stop 
electrons from entering the sheath. Third, the ions are almost collisionless in the sheath. ${ }^{11}$ In this experiment, the maximum sheath extent was $4.5 \mathrm{~cm}$. In comparison, the mean free path ${ }^{38}$ for the ions is $6.0 \mathrm{~cm}$. Fourth, the electrode is planar. (The theory has also been applied to cylindrical and spherical electrodes. ${ }^{8,9}$ ). Fifth, the plasma is $\mathrm{DC}$ and the sheath is pulsed. One difference between our experiment and the theory is the presence of the fast primary and secondary electrons in the discharge. The density of these fast electrons is much less than the bulk of the electrons. However, the ratio of the ion flux to the secondary flux at the cathode is approximately 0.1 . The theoretical work of Howes ${ }^{39}$ suggests that under these conditions, the secondary electrons do not strongly influence the ion density profile in the sheath. The experimental work of Goeckner et al. ${ }^{19}$ indicates that this is also true for the primary electrons.

The predictions of the model and the experimental data show moderate agreement at small sheath widths and disagree at large widths, see Fig. 7. A simulation based on this model was described by Emmert and Henry. ${ }^{8}$ It was formulated to simulate the dynamics of pulsed sheaths used in commercial applications. We have used it here to predict the ion density and the position of the sheath edge. These results are shown in Figs. 6 and 8. The simulation is based on a two-fluid model and requires advanced knowledge of $T_{e}$ and $n_{0}$. For this prediction, we used $T_{e}=0.57$ $\mathrm{eV}$, and $n_{0}=6.25 \times 10^{9} \mathrm{~cm}^{-3}$. Because of the uncertainty of the experimental density measurements, we also used $n_{0}=6.95 \times 10^{9} \mathrm{~cm}^{-3}$ and $5.55 \times 10^{9} \mathrm{~cm}^{-3}$ to produce the error bars on the predictions of the model that are shown in Figs. 7 and 8. These densities result in the "best" agreement at small sheath widths. Higher densities could be used to improve the agreement between the model and the experiment at large sheath widths however, this results in a corresponding increase in the error at small distances.

There are three physical phenomena that are not accounted for in the model that might explain the discrepancies. First, there is no presheath in the model. One can see a manifestation of the presheath in Fig. 5, where the density increases as one moves away from the cathode. This will affect the results because the sheath expansion is faster the lower the plasma density. Thus, at small distances the sheath should expand slightly faster than the model predicts. Looking at Figs. 6-8, one sees that this is observed experimentally. Second, the model is only one dimensional and thus does not account for the two-dimensional nature of the experimental sheath. In the simulation, it is assumed that the cathode is an infinite plane. At distances that are approximately the same as the radius of the cathode, geometric effects become important and the experimental sheath can no longer be described as a planar sheath. ${ }^{9,16}$ In this experiment the radius of the electrode is $6.35 \mathrm{~cm}$, while the maximum sheath width was $\approx 4.5 \mathrm{~cm}$. Thus, while the sheath is still predominately planar in nature, twodimensional effects might begin to be important near the maximum extent of the sheath. The final physical phenomenon not in the model is ion-neutral collisions. Sheridan and Goree ${ }^{11}$ have shown that when the mean free path for the ions is 1.9 times the sheath width, collisions influence the ion energy in the sheath. In our experiment, the mean free path ${ }^{38}$ was approximately $6.0 \mathrm{~cm}$. Thus, one would expect that at widths larger than $3.2 \mathrm{~cm}$ the ion velocity and hence the sheath propagation will be slowed by collisions. This is precisely the distance at which the results of the experiment and the model begin to diverge the most.

Because of these physical phenomena, the collisionless two-fluid model of Widner et al. ${ }^{9}$ and Emmert and Henry ${ }^{8}$ is not well suited for many of the commercial applications of pulsed sheaths. First, we see from the experimental data that the model is not suitable to applications in which the sheath width is greater than half the ions' mean free path. While the plasma density and the neutral pressure employed in this experiment are not unusual in ion implantation devices, the bias used in this experiment is much lower than normal. Typically for the implantation of metal surfaces, the electrode is biased to approximately $-50 \mathrm{kV}$, rather than the $-5 \mathrm{kV}$ used in this experiment. Thus, in a typical commercial application the sheath width might be much wider than the one observed here. Assuming the ion mean free path is similar to the mean free path encountered in this experiment, the maximum sheath width might be several mean free paths wide. Second, we see from the experimental data that the model is not suitable for commercial applications if a substantial presheath exists. Because of the presheath, both the predicted number of ions hitting the electrode and the ion energy profile will be in error. This will result in errors in the predicted depth and dose of the implanted ions.

\section{E. Low voltage pulses}

Widner et al. ${ }^{9}$ examined the propagation of a sheath produced by low-voltage pulses. Like our experiment, they examined an expanding sheath near the center of a biased disk. Their work focused on relatively low-voltage pulses, whereas ours focuses on high-voltage pulses. (The appropriate figure of merit is $e V_{\text {electrode }} / T_{e}$, where $V_{\text {electrode }}$ is the maximum applied bias. For Widner et al. this value is 50 , while for this article the value is 8772 .) Widner et al. found that the sheath initially propagates faster than $C_{s}$ but then slows to approximately $C_{s}$ at large distances. As shown in Fig. 8, our experimental results exhibit similar changes in $V_{s}$ for the $\mathrm{HV}$ pulse. Besides their experimental work, Widner et al. also developed a simulation based on the twofluid model. They found for their experimental conditions, that the two-fluid model provides accurate predictions of the sheath edge. They also measured $n_{e}$ near the sheath edge and again found good agreement with the model. Under the caveats given above, we have found that the model also provides reasonable predictions of $n_{i}$ near the sheath edge. This implies that the model reported by Widner $e t a l$. and later by Emmert and Henry has reasonable predictive capabilities throughout a wide range of applied voltages.

\section{SUMMARY}

We have used LIF to measure the ion density near the edge of an expanding sheath. This expanding sheath was 
produced by applying a HV bias to an electrode that had been immersed in a nitrogen plasma. It was found that the sheath initially expands faster than $C_{s}$ but then slows to approximately $C_{s}$. These experimental data were compared to the predictions of a simulation, which is based on a collisionless two-fluid model of the sheath. When one compares the time-varying location of the sheath edge, fair agreement is found for distances less than 0.5 ion mean free paths from the surface. For distances greater than 0.5 mean free paths, the agreement deteriorates. (The sheath studied in this experiment did not expand beyond 0.75 mean free paths.) This change in the predictive capabilities of the collisionless model is in strong agreement with the DC-collisional model of Sheridan and Goree. In addition, because the model does not include a presheath, it does not accurately predict the ion density profile. Because the model lacks both collisions and a presheath, the model is not well suited to be applied to most ion implantation processes. Finally, we observed that the model does not account for multidinensional affects that occur for large sheath widths. However, by including the data of Widner et al., ${ }^{9}$ we show that the collisionless two-fluid model provides reasonable predictive capabilities throughout a wide range of applied biases, provided one observes the restrictions discussed above.

\section{ACKNOWLEDGMENTS}

We thank Jim Lawler for many helpful discussions. We also thank Nat Horswell and Paul Fetherston for helping us prepare the cathode/stage used in this experiment. Finally, we thank Gil Emmert for supplying us with a copy of his simulation of pulsed sheaths.

This work was supported by Grant No. ECD-8721545 from the National Science Foundation and by contributions from the industrial partners of the Engineering Research Center for Plasma-Aided Manufacturing.

\section{APPENDIX: PLASMA PRODUCTION BY SECONDARY ELECTRONS}

A simple calculation can be used to estimate the plasma produced by the secondary electrons. This calculation requires the knowledge of the lifetimes of the plasma species, from which we can calculate the average number of ions produced by each primary/secondary electron. This in turn will be used to estimate the density of the ionelectron pairs produced by the secondary electrons during the pulse.

The lifetimes of the ions, and primary and secondary electrons can be calculated from the loss rate of each of the species. The particle loss rate for each species can be approximated by

$$
\left.\frac{d N}{d t}\right|_{\text {loss }}=\frac{n V}{\tau} \approx n v A .
$$

Here, $N$ is the total number of the species, $n$ is the density of the species, $v$ is the average velocity of the species, $\tau$ is the average lifetime of the species, $V$ is the plasma volume, and $A$ is the loss area for the species. Rewriting this, we find $\tau$ for each species in terms of the loss area, velocity, and plasma volume

$$
\tau \approx V / v A \text {. }
$$

The velocity can be calculated from the energy/ temperature, while the volume of the plasma can be approximated by the chamber volume. The loss area for each species is the sum of the loss area at the magnetic cusps ${ }^{40}$ and the surface area inside the chamber that is not magnetically shielded. We find for this device that $A_{i}=6760$ $\mathrm{cm}^{2}, A_{p}=6526 \mathrm{~cm}^{2}$, and $A_{s}=9416 \mathrm{~cm}^{2}$ (Here $i, p$, and $s$ stand for the ions; primaries, and secondaries, respectively). Assuming an ion temperature ${ }^{26}$ of $0.03 \mathrm{eV}$, and energies for the primaries and secondaries of 125 and 5000 $\mathrm{eV}$, we find $\tau_{i} \approx 2070 \mu \mathrm{s}, \tau_{p} \approx 0.142 \mu \mathrm{s}$, and $\tau_{s}=0.016 \mu \mathrm{s}$. Without the pulser on the discharge is stable and the loss rate for the ions is balanced by the production rate. Hence

$$
\left.\frac{d N}{d t}\right|_{\text {loss }}=-\left.\frac{d N}{d t}\right|_{\text {production }}=\frac{n V}{\tau} \approx n v A .
$$

By dividing the ion production rate by the $2 \mathrm{~A}$ primary electron current (and the elemental charge) we find that each primary electron produces 0.14 ion-electron pairs. Using the chamber width and the mean free path for ionization, ${ }^{33}$ it is found that the average primary electron must make 2.6 bounces off the walls to create the 0.14 ion-electron pairs. By multiplying these 2.6 bounces by the ratio of the loss areas, we can estimate that the secondary electrons will make 1.79 bounces. Using the mean free path for ionization, ${ }^{34}$ and assuming that most of the current to the stage (see Fig. 2) is due to secondaries, it is found that during the pulse the secondaries produce ion-electron pairs at a rate of $1.72 \times 10^{18} \mathrm{~Hz}$. We note that this is approximately the same as the production rate by the primary electrons, $n_{i} V / \tau_{i} \approx 1.78 \times 10^{18} \mathrm{~Hz}$. However, this production is only for approximately $20 \mu \mathrm{s}$, whereas the primaries produce ion-electron pairs continually. Taking into account the length of the HV pulse, and noting the short lifetime of the secondaries, we find that the secondaries only add $\approx 6 \times 10^{7} \mathrm{~cm}^{-3}$ to the plasma density, or 1 part in 100. Thus, the secondary electrons do not play an important role in plasma production and we do not expect the background plasma to change substantially during the HV pulse.

\footnotetext{
${ }^{1}$ The Collected Works of Irving Langmuir, edited by C. Guy Suits (Pergamon, New York, 1961), Vol. 4, pp. 1-98.

${ }^{2}$ F. F. Chen, in Plastic Diagnostic Techniques, edited by R. II. IIuddlestone and S. L. Leonard (Academic, New York, 1965), pp. 113-200.

${ }^{3}$ A. H. Boozer, Phys. Fluids 19, 1210 (1976).

${ }^{4}$ C. D. Child, Phys. Rev. 32, 492 (1911).

${ }^{5}$ R. J. Procassini, C. K. Birdsall, and E. C. Morse, Phys. Fluids B 2, 3191 (1990).

${ }^{6}$ M. A. Lieberman, IEEE Trans. Plasma Sci. PS-16, 638 (1988).

${ }^{7}$ M. A. Lieberman, J. Appl. Phys. 65, 4186 (1989).

${ }^{8}$ G. A. Emmert and M. A. Henry, J. Appl. Phys. 71, 113 (1992).

${ }^{9}$ M. Widner, I. Alexeff, W. D. Jones, and K. E. Lonngren, Phys. Fluids 13, 2532 (1970).

${ }^{10}$ P. L. Auer, Phys. Fluids 26, 1212 (1981)

${ }^{11}$ T. E. Sheridan and J. Goree, Phys. Fluids B 3, 2796 (1991).

${ }^{12}$ P. D. Goldan, Phys. Fluids 13, 1055 (1970).
} 
${ }^{13}$ M. H. Cho, N. Hershkowitz, and T. Intrator, J. Vac. Sci. Technol. A 6, 2978 (1988).

${ }^{14}$ M. Shamim, J. T. Scheuer, and J. R. Conrad, J. Appl. Phys. 69, 2904 (1991).

${ }^{15}$ M. Shamim, Ph.D. thesis, University of Wisconsin, Madison, 1992.

${ }^{16}$ M. M. Shamim, R. P. Fetherston, K. Sridharan, and J. R. Conrad, Plasma Sources Sci. Technol, 2, 81 (1993).

${ }^{17}$ R. A. Gottscho, R. H. Burton, D. L. Flamm, V. M. Donnelly, and G. P. Davis, J. Appl. Phys. 55, 2707 (1984).

${ }^{18}$ R. A. Gottscho and M. L. Mandich, J. Vac. Sci. Technol. A 3, 617 (1985).

${ }^{19}$ M. J. Goeckner, J. Goree, and T. E. Sheridan, Phys. Fluids B 4, 1663 (1992).

${ }^{20}$ I. Alexeff, W. D. Jones, K. E. Lonngren, and D. Montgomery, Phys. Fluids 12, 345 (1969).

${ }^{21}$ J. R. Conrad, J. L. Radke, R. A. Dodd, F. J. Worzala, and N. C. Tran, J. Appl. Phys. 62, 4591 (1987).

${ }^{22}$ E. C. Jones and N. W. Cheung, IEEE Electron. Device Lett. ED-14, 444 (1993).

${ }^{23}$ J. R. Conrad, R. A. Dodd, S. Han, M. Madapura, J. Scheuer, K. Sridharan, and F. J. Worzala, J. Vac. Sci. Technol. A 8, 3146 (1990).

${ }^{24}$ F. F. Chen, Introduction to Plasma Physics (Plenum, New York, 1974), pp. 244-249.

${ }^{25}$ K. N. Leung, T. K. Samec, and A. Lamm, Phys. Lett. A 34, 490 (1975).

${ }^{26}$ M. J. Goeckner, J. Goree, and T. E. Sheridan, Phys. Fluids B 3, 2913 (1991).

${ }^{27}$ M. J. Goeckner, J. Goree, and T. E. Sheridan, Rev. Sci. Instrum 64, 996 (1993).
${ }^{28}$ This is in contrast to "sub-Doppler" measurements, where the laser's spectral line is narrow. In such cases, the ion velocity distribution function can be ascertained. Such measurements are described in detail in numerous publications. See, for example, Refs. 26 and 27.

${ }^{29}$ J. E. Lawler, W. A. Fitzsimmons, and L. W. Anderson, Appl. Opt. 15, 1083 (1976).

${ }^{30}$ W. J. Alford, Ph.D. thesis, Physics Department, University of Colorado, Boulder, 1984.

${ }^{31}$ B. Y. Tang, R. P. Fetherston, M. Shamim, R. A. Breun, A. Chen, and J. R. Conrad, J. Appl. Phys. 73, 4176 (1993).

${ }^{32}$ M. M. Shamim, and J. T. Scheuer, R. P. Fetherston, and J. R. Conrad, J. Appl. Phys. 70, 4756 (1991).

${ }^{33}$ E. Krishnakumar and S. K. Srivastava, J. Phys. B 23, 1893 (1990).

${ }^{34}$ The data in Ref. 33 extend only to $1 \mathrm{keV}$. To calculate the mean free path for $5 \mathrm{keV}$ electrons, we have extrapolated the data from Ref. 33 by assuming that the ionization rates are constant. This is not unreasonable as the ionization rates found from the data in Ref, 33 are constant from $200 \mathrm{eV}$ to $1 \mathrm{keV}$.

${ }^{35}$ G. M. Barrow, Introduction to Molecular Spectroscopy (McGraw-Hill, New York, 1962), pp. 40-53.

${ }^{36} \mathrm{G}$. A. Collins and $\mathbf{J}$. Tendys, Plasma Source Sci. Technol. (submitted 1993).

${ }^{37} \mathrm{G}$. Herzberg, Molecular Spectra and Molecular Structure I Spectra of Diatomic Molecules, 2nd ed. (Krieger, Malabar, FL, 1989), p. 554.

${ }^{38}$ J. A. Meyer, G.-H. Kim, M. J. Goeckner, and N. Hershkowitz, Plasma Sources Sci. Technol. 1, 147 (1992).

${ }^{39}$ W. L. Howes, J. Appl. Phys. 36, 2039 (1965).

${ }^{40}$ N. Hershkowitz, K. N. Leung and T. Romesser, Phys. Rev. Lett. 35, 277 (1975). 